\title{
ON A THEOREM CONCERNING THE PROLONGATION OF A DIFFERENTIAL STSTEN
}

\author{
YOZÔ MATSUSHIMA
}

E. Cartan has proved that the prolonged system of a Ptaffian system in involution is also in involution." But he has treated only the case of a Pfafian system of some special type. On the other hand in his book [ 3$]$ he has reduced the solution of any differential system to the solution of a Pfaffian system of the type mentioned above, and this reduction is precisely the method of the prolongation of a differential system. ${ }^{2)}$ Therefore it seems to be disirable to establish the above mentioned theorem in the case of an arbitrary differentinl system.

$\S 1$. In this section we mention some definitions and theorems from the theory of differential systems which shall be used later. We use freely the notations and terminologies in Bourbaki [1] and Chevalley [ $\}$ ].

1) Let $M^{n}$ be an analytic manifold of dimension $n$ and let $f_{1}, \ldots f$ s be a system of analytic functions which are defined on a neighbourhood $U$ of a point $a \in M^{n}$ such that $f_{1}(a)=\ldots=f_{s}(a)=0$. The system of equations

$$
f_{1}=0, \ldots, f_{s}=0
$$

is said to be regular of dimension $m$ at the point $a$ if the following conditions are satisfied:

c) There exists a system of coordinates $\left(y^{1}, \ldots, y^{n}\right)$ on $M^{n}$ at the point $a$ and a coordinate neighbourhood $V$ of $a$ with respect to this system of coordinates such that $V \subset U$ and the set of all the points $p \in V$ such that $f_{1}(p)=\ldots$ $=f_{s}(p)=0$ coincides with the set of all the points $p \in V$ such that $y^{m+1}(p)$ $=\ldots=y^{n}(p)=0$;

$\beta$ ) the rank of the differentials $\left(d f_{1}\right)_{a}, \ldots,\left(d f_{s}\right)_{a}$ is equal to $n-m$.

If the system of equations (1.1) satisfies the condition $(\alpha)$ only, we call it semi-regular of dimension $m$ at the point $a$.

If the system of equations (1.1) is regular of dimension $m$ at the point $a$ and if the differentials $\left(d f_{1}\right)_{a} \ldots .\left(d f_{n-m}\right)_{a}$ are linearly independent, then $\left(d f_{i}\right)_{a}(i=n-m+1, \ldots, s)$ are linearly dependent of $\left(d f_{1}\right)_{a} \ldots,\left(d f_{n-m}\right)_{a}$.

Received February 4, 1953.

1) Cartan [2], Chapitre I.

2) See Cartan [3], Chapitre VI. 
2). Let $V^{m}$ be a linear space of dimension $m$ over a field $K$ and $V^{p}$ a subspace of $V^{m}$ of dimension $p$ and let $V^{*^{m}}$ and $V^{* r}$ be the dual spaces of $V^{m}$ and $V^{p}$ respectively. Denote by $\eta_{\text {the }}$ identity mapping of $V^{p}$ into $V^{m}$

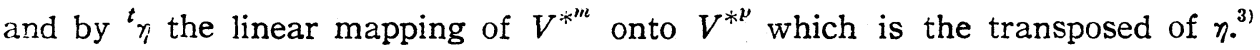
The elements $f^{1}, \ldots, f^{r}$ of $V^{* m}$ are said to be linearly independent on $V^{p}$ if ${ }^{t} \eta\left(f^{1}\right), \ldots,{ }^{t} \eta\left(f^{\boldsymbol{r}}\right)$ are the linearly independent elements of $V^{* \mu}$.

3) Let $D^{n}$ be a domain in the euclidean space of dimension $n$. Let $\Xi$ be a differential system,

$$
\Im:\left\{\begin{array}{cc}
\theta_{\alpha}^{(0)}=0 & \left(\alpha=1, \ldots, u_{0}\right) \\
\theta_{\alpha}^{(1)}=0 & \left(\alpha=1, \ldots, u_{1}\right) \\
\theta_{\alpha}^{(2)}=0 & \left(\alpha=1, \ldots, u_{2}\right) \\
\ldots, \ldots, & \ldots, \ldots, \ldots
\end{array}\right.
$$

where $\theta_{\alpha}^{(r)}$ denote analytic differential forms of order $r$ on $D^{n}$. In particular $\theta_{\alpha}^{(0)}$ are analytic functions on $D^{n}$. We denote also by $\Xi$ the set of the differential forms appearing in the equations (1.2). The differential system $\Xi$ is called closed if $d \theta_{a}^{(r)} \in \Xi$ for any $\theta_{a}^{\left(r^{\prime}\right.} \in \cong$. We may always assume that $\Xi$ is closed.

4) We denote in the following by $E^{p}(a)(p \geqslant 0)$ a contact element of $D^{n}$ of dimension $p$ and of origin $a$. In particular $E^{n}(a)$ is the tangent space of $D^{n}$ at the point $a$ and $E^{0}(a)=a$. Let $E^{0}(a)=a$ be an integral point ( = integral element of dimension 0 ) of the system $\Xi$, i.e. $\theta_{\alpha}^{(0)}(a)=0$. The linear functions on $E^{n}(a)$ defined by

$$
\left\langle L(a), \quad\left(\theta_{\alpha}^{(1)}\right)_{a}\right\rangle^{t^{\prime}}, \quad L(a) \in E^{n}(a), \quad \alpha=1, \ldots, u_{1},
$$

where $(\theta)_{a}$ denotes the value of the differential form $\theta$ at the point $a$, are called the polar functions of the integral point $E^{0}(a)$. We denote by $t\left(E^{0}(a)\right)$ the rank of the polar functions of $E^{0}(a)$. Let $E^{p}(a)(p>0)$ be an integral element of dimension $p$ of the system $\Xi$ and $L_{1}(a) \ldots, L_{p}(a)$ be a base of $E^{p}(a)$. The linear functions on $E^{n}(a)$ defined by

$$
\begin{aligned}
& \left\langle L_{i_{1}}(a) \wedge \ldots \wedge L_{i_{s-1}}(a)_{\wedge} L(a),\left(\theta_{\alpha}^{(s)}\right)_{a}\right\rangle^{4)}, \\
& \left(\begin{array}{l}
L(a) \in E^{n}(a), i_{1}<\ldots<i_{s-1}, \\
\alpha=1, \ldots, u_{s} ; \quad s=1,2, \ldots, p+1
\end{array}\right)
\end{aligned}
$$

are called the polar functions of the integral element $E^{p}(a)$ with respect to the base $L_{1}(a), \ldots, L_{p}(a)$ of $E^{p}(a)$. Denote by $t\left(E^{p}(a)\right)$ the rank of these polar functions. $t\left(E^{p}(a)\right)$ is independent of the choice of the base of $E^{p}(a)$.

5) Let $E^{p}(a)$ be an integral element of the system $\widetilde{\Xi}$ and let

3) Bourbaki [1], Chapitre II, p. 56 .

4) For the notation $\langle,>$ and $\wedge$, see Bourbaki [1], Chapitre II, p. 42 and Chapitre III $\$ 5$. Notice that the value of a differential form of order $r$ at a point $a$ is an element of $\wedge r E(a)^{* n}$, where $E(a)^{* n}$ denotes the dual space of $E^{n}(a)$. See Chevalley [4]. 


$$
t_{\nu}=\operatorname{Max}_{\left.F^{\nu}, a \in E v_{(}\right)} t\left(E^{\nu}(a)\right) \quad(\nu=0,1,2, \ldots, p-1) .
$$

Then it can be proved that $t_{0} \leqq t_{1} \leqq \ldots \leqq t_{p-1}$ and that there exists a chain $E^{0}(a) \subset E^{1}(a) \subset \ldots \subset E^{p-1}(a) \subset E^{p}(a)$ of subspaces of $E^{p}(a)$ such that $t_{\text {v }}$ $=t\left(E^{\prime}(a)\right)$ for $\nu=0,1, \ldots, p-1$. Let

$$
s_{0}=t_{0}, \quad s_{\nu}=t_{\nu}-t_{\nu-1} \geqslant 0 \quad(\nu=1,2, \ldots, p-1) .
$$

The integers $s_{0}, \ldots, s_{p-1}$ are called the characters of the integral element $E^{p}(a)$ :

If $E^{p}(a)$ is an ordinary integral element ${ }^{6}$ of the system $\equiv$ and if $E^{0}(a)$ $\subset E^{1}(a) \subset \ldots \subset E^{p-1}(a) \subset E^{p}(a)$ is a chain of the regular integral elements ${ }^{6}$ contained in $E^{p}(a)$, then $t_{\nu}=t_{\nu}\left(E^{\nu}(a)\right)(\nu=0,1, \ldots, p-1)$.

6) Let $M^{n}$ be an analytic manifold of dimension $n$ and $S_{n, p}\left(M^{n}\right)$ the analytic manifold of all the sets of $p$ lineariy independent tangent vectors $(p$ frames) of $M^{n}$ and $G_{n, p}\left(M^{n}\right)$ the analytic manifold of all the contact elements of dimension $p$ of $M^{n}$. Let $\pi\left(E^{p}(r)\right)=r$ for $E^{p}(r) \in G_{n, p}\left(M^{n}\right)$. Then the mapping $\pi$ is an analytic mapping of $G_{n, p}\left(M^{n}\right)$ onto $M^{n}$.

Let $v^{p}(r)=\left(L_{1}(r), \ldots, L_{p}(r)\right) \in S_{n, p}\left(M^{n}\right)$, where $L_{i}(r)$ are the linearly independent tangent vectors of origin $r$ and let $E^{t}(r)=\left\{L_{1}(r), \ldots, L_{p}(r)\right\}$ be the contact element of dimension $p$ spanned by $L_{1}(r), \ldots, L_{p}(r)$. Then the mapping $\alpha\left(v^{p}(r)\right)=E^{p}(r)$ is an analytic mapping of $S_{n, p}\left(M^{n}\right)$ onto $G_{n, p}\left(M^{n}\right)$. Let $\mathbf{U}$ be a neighbourhood of $E^{p}(a)$ in the manifold $\mathrm{G}_{n, p}\left(M^{n}\right)$ and $\psi$ an analytic mapping of $\mathbf{U}$ into $S_{n, p}\left(M^{n}\right)$. The mapping $\psi$ is called an analytic cross section defined on $\mathbf{U}$ if

$$
\alpha \circ \psi\left(E^{p}(r)\right)=E^{p}(r)
$$

5) In the case where $\left(d x^{1}\right)_{a} \ldots,\left(d x^{p}\right)_{a}$ are linearly independent on $E^{n}(a)$ the values of the characters of $E^{n}(a)$ coincide with those of "caractères reduits." See Cartan [3], p. 90.

6) For the definition of ordinary and regular integral elements of a differential system, see Cartan [3], Chapitre IV. The definition of regular integral element in Kähler [5], p. 23 is different from that of Cartan. It follows that a regular integral element in the sense of Cartan is also regular in the sense of Kähler. Cf. Schouten and Kulk [6], Chap. VIII.

We give here the definition of regular element in the sense of Kähler using our notations. An integral element of the system (1.2) is called regular in the sense of Kähler, if the following conditions are satisfied;

(x) The systems of equations

$$
\begin{aligned}
& H_{0}^{\alpha}\left(E^{p}(r)\right)=0 \quad\left(\alpha=1, \ldots, u_{0}\right) \\
& H_{\alpha i_{1}}^{(s)} \ldots i_{s}\left(E^{p}(r)\right)=0 \quad\left(\alpha=1, \ldots, u_{s}, s=1,2, \ldots, 1 \leqq i_{1}<\ldots<i_{s} \leqq p\right)
\end{aligned}
$$

are regular at the point $E^{p}(a)$ respectively (cf. $\left.(1.7),(1.8)\right)$;

$(\beta)$ There exists a neighbourhood $\mathbf{V}$ of the point $E^{p}(a)$ in the manifold $G_{n, p}\left(D^{n}\right)$ such that, if $E^{\nu}(r)$ is an integral element of the system (1.2) which is contained in $\mathbf{V}$, then $t\left(E^{p}(a)\right) \geqslant t\left(E^{p}(r)\right)$.

It must be noted that the rank of the differentials of the functions $H_{\alpha i_{1}}^{(s)} \ldots i_{s}$ at $E^{p}(a)$ is independent of the choice of the analytic cross section $\psi$ which is used to define the functions $H_{\alpha i_{1}}^{\prime s} \ldots \iota^{\prime}$ (cf. 6$)$ ). 
for any $E^{p}(r) \in \mathrm{U}$. Now let $M^{n}=D^{n}$ and $\varphi^{\prime}\left(E^{p}(r)\right)=v^{p}(r)=\left(L_{1}(r), \ldots L_{p}(r)\right)$. We define the analytic functions on $\mathbf{U}$ as follows:

$$
\begin{gathered}
H_{\alpha}^{(0)}\left(E^{p}(r)\right)=\left(\theta_{\alpha}^{(0)} \circ \pi\right)\left(E^{p}(r)\right) \\
H_{\alpha i_{2}}^{(s)} \ldots i_{s}\left(E^{p}(r)\right)=\left\langle L_{i_{\mathrm{i}}}(r) \wedge \ldots \wedge L_{t_{s}}(r), \theta_{\alpha}^{(s)}(r)\right\rangle, \\
\left(\begin{array}{l}
\alpha=1,2, \ldots, u_{s} ; s=1,2, \ldots ; \\
1 \leqq i_{1}<i_{2}<\ldots<i_{s} \leqq p
\end{array}\right),
\end{gathered}
$$

where $\theta_{\alpha}^{(k)} \in \cong$.

A contact element $E^{p}(\boldsymbol{r})$ in $\mathbf{U}$ is an integral element of the system $\Xi$ if and only if all the functions (1.7), (1.8) vanish at $E^{p}(r)$.

Suppose that $E^{p}(a)$ is an integral element of the systems $\cong$. Then it can be proved easily that the rank of the differentials of the functions (1.8) at the point $E^{p}(a)$ is independent of the choice of the analytic cross section $\psi$ defined in a neighbourhood of $E^{p}(a) .^{7)}$

7) Let $E^{p}(a)$ be an integral element of the system $\cong$. Then $E^{p}(a)$ is ordinary if and only if the following two conditions are satisfied;

1) the system of equations $H_{\alpha}^{(0)}=0$ is regular of dimension $\nu_{0}$ at the point $E^{p}(a)$;

2) the system of equations $H_{\alpha}^{(0)}=0, H_{\alpha i_{1}}^{(S)} \ldots i_{s}=0$ is regular of dimension $\nu_{0}+M$ at the point $E^{p}(a)$, where

$$
M=p(n-p)-\left[p s_{0}+(p-1) s_{1}+\ldots+s_{p-1}\right]
$$

and $s_{i}$ are the characters of the integral element $E^{p}(a){ }^{s)}$

8) Let $E^{p}(a)$ be an integral element of the system $\Im$ and let $s_{0}, \ldots, s_{p-1}$ be the characters of $E^{p}(a)$. Then there exist at least $p s_{0}+(p-1) s_{1}+\ldots+s_{p-1}$ functions (1.8) whose differentials at the point $E^{p}(a)$ are linearly independent. We may choose these $p s_{0}+(p-1) s_{1}+\ldots+s_{p-1}$ functions in the following way. Let $\left(x^{1}, \ldots, x^{n}\right)$ be a system of coordinates defined on $D^{n}$. We may assume that the differentials $\left(d x^{1}\right)_{a} \ldots,\left(d x^{p}\right)_{a}$ are linearly independent on $E^{p}(a)$. Let $E^{p}(a) \supset E^{p-1}(a) \supset \ldots \supset E^{1}(a) \supset E^{0}(a)=a$ be a chain of subspaces of $E^{p}(a)$ such that $t\left(E^{\nu}(a)\right)=t_{\nu}=s_{0}+\ldots+s_{\nu}\left(\nu=0,1, \ldots, p-1\right.$. Replacing $x^{1}, \ldots$. $x^{p}$ by a suitable linear combinations with constant coefficients, if necessary, we may assume that

$$
\begin{aligned}
E^{\nu}(a)=\left\{L \in E^{p}(a) \mid\left\langle L,\left(d x^{\nu+1}\right)_{a}\right\rangle=\right. & \left.\ldots=\left\langle L,\left(d x^{p}\right)_{a}\right\rangle=0\right\} \\
& (\nu=1,2, \ldots p-1) .
\end{aligned}
$$

i) Let i, be an another cross section defined on $\mathbf{U}$. We can define analogously the functions $H_{\alpha i_{1}}^{(s)} \ldots i_{s}$ by using the cross section $\bar{\psi}$. Then

$$
\bar{H}_{\alpha i_{1}}^{(s)} \ldots i_{s}=\underset{j_{1}<\ldots<j s}{\Sigma} F_{j_{1}} \ldots j_{s} \cdot H_{\alpha j_{1}}^{(s)} \ldots j_{s},
$$

where $F_{j_{1}} \ldots j_{s}$ are analytic functions on $\mathbf{U}$.

8) Cartan [3], p. 91 and Kähler [5], pp. 40-44. 
Let $\psi_{0}$ be the analytic cross section defined on $D^{x}$ by (1.15) and (1.16) (see 9 ) below). The tangent vectors $L_{1}(a), \ldots, L_{p}(a)$ defined by (1.16) form a base of $E^{p}(a)$ such that $L_{1}(a), \ldots, L_{\nu}(a)$ spann the subspace $E^{\nu}(a)$. We define the polar functions of $E^{p}(a)$ with respect to this base by (1.4). Then the linear functions (1.4) such that $s=1$ or $i_{1}<\ldots<i_{s-1} \leqq \nu$ in case $s>1$ are the polar functions of $E^{2}(a)$ with respect to the base $L_{1}(a), \ldots, L_{2}(a)$. Since $t_{2}=t_{2}\left(E^{2}(a)\right)$ we may choose $t_{p-1}=s_{0}+\ldots+s_{p-1}$ linearly independent polar functions $F_{1}(L(a)$. $\ldots, F_{t^{\prime-1}}(L(a))$ in such a way that $F_{1}, \ldots F_{t,}$ are the polar functions of $E^{\nu}(a) . \quad(\nu=0,1, \ldots, p-1)$. Let $i \leqq t$, and let

$$
\begin{aligned}
F_{i}(L(a))=\left\langle L_{j_{\mathrm{i}}}(a) \wedge \cdots \wedge\right. & \left.L_{j_{s-1}}(a) \wedge L(a),\left(\theta_{\alpha}^{(s)}\right)_{a}\right\rangle \\
& \left(j_{1}<\ldots<j_{s-1} \leqq \nu\right) .
\end{aligned}
$$

Let

$$
H_{i}^{(\nu+1)}\left(E^{p}(r)\right)=\left\langle L_{j_{1}}(\boldsymbol{r})_{\wedge} \ldots \wedge L_{j_{s-1}}(r)_{\wedge} L_{s+1}(r),\left(\theta_{\alpha}^{(s)}\right)_{r}\right\rangle,
$$

where $L_{j}(r)$ are defined by (1.16).

Thus for each $F_{i}, i \leqq t_{\nu}$, we obtain an analytic function $H_{i}^{(3+1)}$ in (1.8) defined on $\mathfrak{D}^{N}$. Then

$$
H_{i}^{(\nu+1)} \quad\left(i \leqq t_{\nu}, \nu=0,1, \ldots, p-1\right)
$$

are the disired functions.

Proof. By (1.11) $\left(d H_{i}^{(\nu+1)}\right)_{F^{y}(a)}$ are linear combinations of the differentials $\left(d x^{j}\right)_{F^{\prime \prime}(a)}(j=1, \ldots, n)$ and $\left(a l_{k}^{\lambda}\right)_{E^{\prime}(a)}$ such that $k \leqq \nu+1$. Let

$$
\left(d H_{i}^{(\nu+1)}\right)_{E^{p}(a)}=\Omega_{i}^{(\nu+1)}+\Omega_{i}^{(\nu+1)},
$$

where $\Omega_{i}^{\prime(\nu+1)}$ are the linear combinations of $\left(d x^{j}\right)_{F^{\prime}}{ }^{\prime}(a)$ and $\left(d l_{k}^{\lambda}\right)_{F^{\prime \prime}(a)}$ such that $k<\nu+1$ and $\Omega_{i}^{(\nu+1)}$ are the linear combinations of $\left(d l_{\nu+1}^{\lambda}\right)_{E^{\nu}(\alpha)}$. Then

$$
\Omega_{i}^{(\nu+1)}=\sum_{\lambda=p+1}^{n}\left\langle L_{j_{1}}(a) \wedge \cdots \wedge L_{j_{s-1}}(a) \wedge\left(\frac{\partial}{\partial x^{\lambda}}\right)_{a},\left(\theta_{\alpha}^{(S)}\right)_{a}\right\rangle\left(d l_{\nu+1}^{\lambda}\right)_{F^{p}}(a) .
$$

Hence by (1.10),

$$
\Omega_{i}^{(\nu+1)}=\sum_{\lambda=p+1}^{n} F_{i}\left(\left(\frac{\partial}{\partial x^{\lambda}}\right)_{a}\right)\left(d l_{\nu+1}^{\lambda}\right)_{F^{p}(a)} .
$$

We show that $\Omega_{i}^{(\nu+1)}\left(\nu=0, \ldots, p-1, i=1, \ldots, t_{\nu}\right)$ are linearly independent. Since the differentials $\left(d l_{\nu+1}^{\lambda}\right)_{E^{v}}(a)$ are linearly independent it is clearly sufficient to show that $\Omega_{1}^{(\nu+1)}, \ldots \Omega_{t}^{(\nu+1)}$ are linearly independent. We apply Lemma 2 , $\S 2$ in the case where $m=n, V^{m}=E^{n}(a), V^{p}=E^{p}(a), f^{i}=\left(d x^{i}\right)_{a}$ and the set $\left(g^{1}, \ldots, g^{s}\right)$ is equal to the set $\left(F_{1}, \ldots, F_{i_{\nu}}\right)$. Since the subspace $E^{n-p}(a)$ of the tangent space $E^{n}(a)$ consisting of all the $L(a) \in E^{n}(a)$ such that $\langle L(a)$, $\left.\left(d x^{i}\right)_{a}\right\rangle=0(i=1,2, \ldots, p)$ is spanned by $\left(\frac{\partial}{\partial x^{\lambda}}\right)_{a}$ and since $F_{1} \ldots . F_{t_{\nu}}$ are linearly independent, we see easily that $\Omega_{1}^{(2+1)}, \ldots \Omega_{t_{\nu}}^{(\nu+1)}$ are linearly inde- 
pendent. Since $\Omega_{i}^{(\nu+1)}$ are linearly independent, it is clear that $\left(d H_{i}^{(\nu+1)}\right)_{E^{p}(a)}$ are linearly independent, q.e.d.

It must be noted that if $\sum_{i=1}^{t v} \lambda^{i}\left(d H_{i}^{(\nu+1)}\right)_{E^{p}(a)}$ is a linear combination of $\left(d x^{j}\right)_{E^{p}(a)}$ and $\left(a l_{k}^{i}\right)_{E^{p}(a)}$ such that $k<\nu+1$, then $\lambda^{i}=0$. This follows from the linear independence of $\Omega_{i}^{(v+1)}$.

In particular if $E^{p}(a)$ is an ordinary integral element of the system $\subseteq$, then any $\left(d H_{\alpha i_{1} \ldots i_{\mathrm{s}}}^{(s)}\right)_{E^{p}(a)}$ is a linear combination of $\left(d H_{i}^{(\nu+1)}\right)_{E^{1}(a)}$ modulo $\left(d H_{\alpha}^{(0)}\right)_{F^{y}(a)}$.

We use these properties of the functions $H_{i}^{(\nu+1)}$ in the proof of Lemma $1, \S 2$.

9) We denote in the following by $\left(x^{1}, \ldots, x^{n}\right)$ a system of coordinates defined on $D^{n}$. Let $\mathfrak{D}^{N}$ be the subspace of $G_{n, p}\left(D^{n}\right)$ consisting of all the contact elements of dimension $p$ on which the differentials $\left(d x^{1}\right)_{a}, \ldots,\left(d x^{p}\right)_{a}$ are linearly independent, where

$$
N=n+p(n-p)
$$

is the dimension of the manifold $G_{n, p}\left(D^{n}\right)$. Then $\mathfrak{D}^{N}$ is a connected open set of $G_{n, p}\left(D^{n}\right)$ and analytically homeomorphic with the manifold $D^{n} \times R^{p(n-p)}$, where $R^{p(n-p)}$ denotes the euclidean space of dimension $p(n-p)$.

Let $E^{p}(r) \in \mathbb{D}^{N}$. Then there exists a system of $p(n-p)$ real numbers $l_{i}^{\lambda}\left(E^{p}(r)\right)(\lambda=p+1, \ldots, n, i=1, \ldots, p)$ uniquely determined by $E^{p}(r)$ such that

$$
{ }^{t} \eta_{r}\left(\left(d x^{\lambda}\right)_{r}\right)=\sum_{i=1}^{p} l_{i}^{\lambda}\left(E^{p}(r)\right)^{t} \eta_{r}\left(\left(d x^{i}\right)_{r}\right),
$$

where $\eta_{r}$ denotes the identity mapping of $E^{p}(r)$ into the tangent space $E^{n}(r)$. Then $l_{i}^{\lambda}$ are analytic functions on $\mathfrak{D}^{N}$ and

$$
\begin{array}{r}
E^{p}(r)=\left\{L \in E^{n}(r) \mid\left\langle L,\left(d x^{\lambda}\right)_{r}-\sum_{i=1}^{p} l_{i}^{\lambda}\left(E^{p}(r)\right)\left(d x^{i}\right)_{r}\right\rangle=0,\right. \\
\lambda=p+1, \ldots, n\}
\end{array}
$$

Let $\pi\left(E^{p}(r)\right)=r$ for each $E^{p}(r) \in \mathfrak{D}^{N}$ and let

$$
X^{k}=x^{k} \circ \quad(k=1, \ldots, n) \text {. }
$$

Then the functions $X^{1}, \ldots, X^{n}, l_{i}^{\lambda}(\lambda=p+1, \ldots, n ; i=1, \ldots, p)$ constitute a system of coordinates on $\mathfrak{D}^{N}$, and the mapping of $\mathfrak{D}^{N}$ into $S_{n, p}\left(D^{n}\right)$ defined by

$$
\psi_{0}\left(E^{p}(r)\right)=\left(L_{1}(r), \ldots, L_{p}(r)\right)
$$

where

$$
L_{i}(r)=\left(\frac{\partial}{\partial x^{i}}\right)_{r}+\sum_{\lambda=p+1}^{n} l_{i}^{\lambda}\left(E^{p}(r)\right)\left(\frac{\partial}{\partial x^{\lambda}}\right)_{r} \quad(i=1, \ldots, p)
$$

is an analytic cross section defined on $\mathscr{D}^{N}$. Hence we can define by using the 
cross section $\psi_{0}$ the functions (1.7) and (1.8) which are analytic on $\mathfrak{D}^{N}$.

Now we consider a closed differential system $\widetilde{\subseteq}$ defined on $\mathfrak{D}^{v}$,

$$
\widetilde{\Im}: \begin{cases}H_{\alpha}^{(0)}=0, \quad d H_{\alpha}^{(0)}=0, & \left(\alpha=1, \ldots, u_{0},\right) \\
H_{\alpha i_{1}}^{(s)} \ldots i_{s}=0, \quad d H_{\alpha i_{2}}^{(s)} \ldots i_{s}=0, & \left(\begin{array}{l}
\left.\alpha=1, \ldots, u_{s}, s=1,2, \ldots\right) \\
1 \leqq i_{1}<\ldots<i_{s} \leqq p,
\end{array}\right. \\
d X^{\lambda}-\sum_{i=1}^{p} l_{i}^{\lambda} d X^{i}=0 & \\
\sum_{i=1}^{p} d l_{i}^{\lambda} d d X^{i}=0 & \end{cases}
$$

The differential system $\widetilde{\subseteq}$ is called the (total) prolongation of the differential system $\mathfrak{S}$.

10) Let $E^{p}(a)$ be an ordinary integral element of the system $\subseteq$ such that $E^{p}(a) \in \mathfrak{D}^{N}$. Then there exists at least an integral element of dimension $p$ and of origin $E^{p}(a)$ of the prolonged system $\widetilde{\subseteq}$ on which $\left(d X^{1}\right)_{E^{\prime}}{ }(a), \ldots,\left(d X^{p}\right)_{F^{p}}(a)$ are linearly irdependent. For, by the existence theorem of Cartan-Kähler ${ }^{2}$ there exists an integral manifold $M^{p}$ of the system $\subseteq$ containing the point $a$ such that the tangent space to the manifold $M^{p}$ at the point $a$ is $E^{p}(a)$. Since $\left(d x^{1}\right)_{a}, \ldots,\left(d x^{p}\right)_{a}$ are linearly independent on $E^{p}(a)$, we can represent the manifold $M^{p}$ in a neighbourhocd of the point a by a system of equations

$$
x^{\lambda}=\varphi^{\lambda}\left(x^{1}, \ldots, x^{p}\right), \quad(\lambda=p+1, \ldots, n),
$$

where $\varphi^{\lambda}$ are the analytic functions defined in a neighbourhood of $\left(x^{1}(a), \ldots\right.$, $\left.x^{p}(a)\right)$.

Then the system of equations

$$
\begin{aligned}
& X^{\lambda}=\varphi^{\lambda}\left(X^{1}, \ldots, X^{p}\right) \\
& l_{i}^{\lambda}=\frac{\partial \varphi^{\lambda}}{\partial X^{i}}\left(X^{1}, \ldots, X^{p}\right), \quad(\lambda=p+1, \ldots, n, \quad i=1, \ldots, p)
\end{aligned}
$$

represents clearly an integral manifold $\mathfrak{M}^{p}$ of the prolonged system (1.19) containing the point $E^{p}(a)$. Then the tangent space to the manifold $\mathfrak{M}^{p}$ at the point $E^{p}(a)$ is clearly an integral element of the prolonged system $\widetilde{\Xi}$ on which $\left(d X^{1}\right)_{E^{p}}(a), \ldots,\left(d X^{p}\right)_{E^{p}}{ }_{(a)}$ are linearly independent.

Definition. ${ }^{10)}$. The differential system (1.2) is called in involution with respect to the variables $x^{1}, \ldots, x^{p}$, if there exists an ordinary integral element $E^{p}(a)$ of the system on which $\left(d x^{1}\right)_{a}, \ldots,\left(d x^{p}\right)_{a}$ are linearly independent.

$\S 2$. We denote in the following by $\xi^{p}\left(E^{p}(r)\right)$ a contact element of the manifold $\mathfrak{D}^{N}$ of dimension $p$ and of origin $E^{p}(r)$ and by $\mathfrak{L}\left(E^{p}(r)\right)$ the tangent vectors of $\mathfrak{D}^{N}$ of origin $E^{p}(\boldsymbol{r})$.

9) Kähler [5], p. 26, Cartan [3], Chapitre IV, Schouten and Kulk [6], Chapter VIII.

10) Cartan [3], p. 89. 
THEOREM 1. Let $E_{0}^{p}\left(a_{0}\right)$ be an ordinary integral element of dimension $p$ and of origin $a_{0}$ of the system (1.2) on which $\left(d x^{1}\right)_{a_{0}}, \ldots,\left(d x^{p}\right)_{a_{0}}$ are linearly independent and let $E_{0}^{p}\left(E_{0}^{p}\left(a_{0}\right)\right)$ be an integral element of dimension $p$ and of origin $E_{0}^{p}\left(a_{0}\right)$ of the prolonged system (1.17) on which $\left(d X^{1}\right)_{E_{0}}^{p_{\left(a_{0}\right)}}, \ldots,\left(d X^{p}\right)_{F_{0}^{y}\left(a_{0}\right)}$ are linearly independent. Then $c_{0}^{p}\left(E_{0}^{p}\left(a_{0}\right)\right)$ is oidivary. Let $s_{0}, \ldots, s_{p-1}$ and $S_{0} \ldots, S_{p-1}$ be the characters of $E_{0}^{p}\left(a_{0}\right)$ and $E_{0}^{p}\left(E_{0}^{p}\left(a_{0}\right)\right)$ respectively. Then

$$
\begin{aligned}
& S_{0}=(p+1) s_{0}+p s_{1}+\ldots+s_{p}, \\
& S_{1}=s_{\nu}+s_{\nu+1}+\ldots+s_{p}, \quad(\nu=1,2, \ldots, p-1),
\end{aligned}
$$

where

$$
s_{p}=(n-p)-\left(s_{0}+\ldots+s_{p-1}\right) .
$$

From Theorem 1 and $\S 1,10)$ we obain immediately

THEOREM 2. If the differential system (1.2) is in involution with respect to the variables $x^{1}, \ldots, x^{p}$, then the prolonged system (1.17) is in involution with respect to the variables $X^{1}, \ldots, X^{p}$.

The remaining part of this paper is devoted to the proof of Theorem 1.

Let $G_{N}, p\left(\mathfrak{D}^{N}\right)$ be the analytic manifold of all the contact elements of $\mathfrak{D}^{N}$ of dimension $p$ and let $\widetilde{D}$ be the subspace of $G_{N, p}\left(\mathbb{D}^{N}\right)$ consisting of all the contact element of dimension $p$ on which $d X^{1}, \ldots, d X^{p}$ are linearly independent. Then $\tilde{D}$ is a connected open set of $G_{N, p}\left(\mathfrak{D}^{N}\right)$ and analytically homeomorphic with the manifold $\mathfrak{D}^{N} \times R^{p(N-p)}$, where $R^{p(N-p)}$ denotes the euclidean space of dimension $p(N-p)$. We may define as in $\S 1,8)$ the analytic functions $t_{i}^{\lambda}, t_{k i}^{\lambda}$ $(\lambda=p+1, \ldots, n ; i, k=1, \ldots, p)$ on $\tilde{\mathfrak{D}}$ as follows. Let $\eta$ be the identity mapping of $\mathscr{F}^{p}\left(E^{p}(r)\right)$ into the tangent space $\mathscr{F}^{N}\left(E^{p}(\boldsymbol{r})\right)$, where $E^{p}(\boldsymbol{r}) \in \mathfrak{D}^{v}$. Then

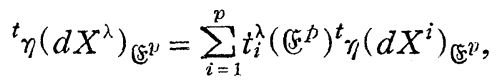

$$
\begin{aligned}
& { }^{t} \eta\left(d l_{k}^{\lambda}\right)_{\mathfrak{G}^{2}}=\sum_{i=1}^{p} t_{k i}^{\lambda}\left(\mathfrak{ङ}^{p}\right)^{t} \eta\left(d X^{i}\right)_{\mathfrak{E}^{p}},
\end{aligned}
$$

where $\mathbb{E}^{p}=\mathbb{S}^{p}\left(E^{p}(r)\right)$.

The mapping $\Psi_{0}$ of $\widetilde{\mathfrak{D}}$ into $S_{x, p}\left(\mathfrak{D}^{x^{x}}\right)$ defined by

$$
\Psi_{n}\left(\mathfrak{S}^{\not}\left(E^{p}(r)\right)=\mathfrak{S}^{p}\left(E^{p}(r)\right)=\left(\Omega_{1}\left(E^{p}(r)\right), \ldots, \mathfrak{Q}_{p}\left(E^{p}(r)\right)\right),\right.
$$

where

$$
\begin{aligned}
& \mathfrak{S}_{i}\left(E^{p}(r)\right)=\left(\frac{\partial}{\partial X^{i}}\right)_{F^{p}(r)}+\sum_{\lambda=p^{\prime}+1}^{n} t_{i}^{\lambda}\left(\xi^{p}\left(E^{p}(r)\right)\left(\frac{\partial}{\partial X^{\lambda}}\right)_{E^{n^{\prime}}(r)}\right. \\
& +\sum_{\lambda=p^{\prime}+1}^{n} \sum_{k=1}^{n} t_{k i}^{\lambda}\left(\xi^{p}\left(E^{p}(r)\right)\left(\begin{array}{c}
\partial \\
\partial t_{k}^{\lambda}
\end{array}\right)_{E^{\prime}(r)}\right.
\end{aligned}
$$

is an analytic cross section defined on $\widetilde{\mathfrak{S}}$.

Further let $I I\left(\widetilde{F}^{p}\left(E^{p}(r)\right)=E^{p}(r)\right.$. Making use of $I I$ and $\Psi_{0}$ we define the analytic functions on $\widetilde{D}$ corresponding to (1.7) and (1.8), 


$$
\begin{aligned}
& H_{a}^{(0)} \circ \Pi=0, \quad\left(\alpha=1, \ldots, u_{0}\right), \\
& H_{\alpha i_{1} \ldots i_{s}}^{(s)} \circ I I=0 \quad\left(\begin{array}{l}
\left.\alpha=1, \ldots, u_{s,}, s=1,2, \ldots,\right) \\
1 \leqq i_{1}<\ldots<i_{\varsigma} \leqq p,
\end{array}\right) \\
& \left\langle\mathcal{Q}_{i}\left(E^{p}(\boldsymbol{r})\right),\left(d H_{\alpha}^{(0)}\right)_{F^{2}{ }^{\prime}(r)}\right\rangle=0 \\
& \left\langle Q_{i}\left(E^{p}(r)\right),\left(d H_{\alpha i_{1}}^{(S)} \ldots i_{s}\right)_{F^{*}}(r)\right\rangle=0 \\
& \left\langle\mathbb{Q}_{i}\left(E^{p}(r)\right),\left(d X^{\lambda}\right)_{E^{\prime}{ }^{\prime}(r)}-\sum_{k=1}^{p} l_{k}^{2}\left(E^{p}(r)\right)\left(d X^{k}\right)_{r^{\prime}{ }^{\prime}(r)}\right\rangle=0
\end{aligned}
$$

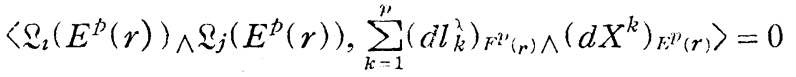

Since $E_{0}^{p}\left(a_{0}\right)$ is ordinary, the systems of equations $H_{\alpha}^{(0)}=0, H_{\alpha i_{1}}^{(s)} \ldots i_{s}=0$ is regular of dimension $\nu_{0}+M$ at the point $E_{0}^{p}\left(a_{0}\right)$. Hence the system of equations (2.5) is regular of dimension $\nu_{0}+M$ at the point $\left[f_{0}^{p}\left(E_{0}^{p}\left(a_{0}\right)\right)\right.$. To prove that $\left(\xi_{0}^{p}\left(E_{0}^{p}\left(a_{0}\right)\right)\right.$ is ordinary, it is sufficient to show that the system of equations $(2.5)$

... (2.9) is regular of dimension $\nu_{0}+M+\tilde{M}$, where

$$
\tilde{M}=p(N-p)-\left[p S_{0}+(p-1) S_{1}+\ldots+S_{p-1}\right] .
$$

Let $E^{p}(a)$ be an integral element of the system (1.2) which is in a suf ficiently small neighbourhood of $E_{0}^{p}\left(a_{0}\right)$. Then $E^{t}(a)$ is ordinary and the values of the characters of $E^{p}(a)$ are the same with those of $E_{0}^{p}\left(a_{0}\right)$. Further since there exists always at least an integral element of the system (1.17) whose origin is $E^{p}(a)$ and on which $d X^{1}, \ldots, d X^{p}$ are linearly independent and since the functions which appear in the left sides of the equations are linear in the variables $t_{i}^{\lambda}=t_{i}^{\lambda}\left(\xi^{p}\left(E^{p}(r)\right), t_{k i}^{\lambda}=t_{k i}^{\lambda}\left(\sum^{p}\left(E^{p}(r)\right)\right.\right.$, it is sufficient to show that the rank of the linear forms in the $p(N-p)$ variables $t_{i}^{\lambda}, t_{i j}^{\lambda}$ which are defined as follows is equal to $p S_{0}+(p-1) S_{1}+\ldots+S_{1-1.1}$.

$$
\begin{aligned}
& \sum_{\lambda=p+1}^{n} t_{i}^{\lambda}\left\langle\left(\begin{array}{c}
\partial \\
\partial \ddot{X}^{\lambda}
\end{array}\right)_{F^{*}(a)}, \quad\left(d H_{\alpha}^{(0)}\right)_{F^{\prime}(a)}\right\rangle \\
& \left(\alpha=1, \ldots, u_{0}, \quad i=1, \ldots p\right) \\
& \sum_{\lambda=p+1}^{n} t_{i}^{\lambda}\left\langle\left(\frac{\partial}{\partial X^{\lambda}}\right)_{F^{\nu}(a)}, \quad\left(d H_{a i_{1}}^{(s)} \ldots i_{s}\right)_{E^{\nu}(a)}\right\rangle \\
& +\sum_{\lambda=p+1}^{n} \sum_{k=1}^{p} t_{k l}^{\lambda}\left\langle\left(\frac{\partial}{\partial l_{k}^{(}}\right)_{F}{ }^{(a)}, \quad\left(d H_{\alpha i_{1}}^{(c)} \ldots i_{s}\right)_{E^{p}(a)}\right\rangle \\
& \left(\begin{array}{l}
\alpha=1, \ldots, u_{s}, \quad s=1,2, \ldots \\
1 \leqq i_{1}<\ldots<i_{s} \leqq p, \quad i=1,2, \ldots, p
\end{array}\right) \\
& t_{i}^{\lambda} \quad(\lambda=p+1, \ldots, n, \quad i=1, \ldots p) \\
& t_{i, j}^{\lambda}-t_{j i}^{\lambda} \quad(\lambda=\mathrm{p}+1, \ldots, n, \quad 1 \leqq i<j \leqq p)
\end{aligned}
$$

Lemma 1. The rank of the linear forms (2.10), (2.11), (2.12) and (2.13) is equal to $\frac{p(\dot{p}+1)}{2}(n-p)+t_{0}+2 t_{1}+\ldots+p t_{p-1}$, where $t_{\text {. }}=s_{0}+\ldots+s_{\text {, }}$. 
Proof. We first notice that the characters of $E^{p}(a)$ are $s_{0}, \ldots, s_{p-1}$. We choose $p s_{0}+(p-1) s_{1}+\ldots+s_{p-1}$ functions $H_{i}^{(\nu)}\left(\nu=1,2, \ldots, p-1 ; i \leqq t_{\nu-1}\right)$ from the system of functions $H_{\alpha i_{1} \ldots i_{s}}^{(s)}$ as in $\left.\S 1,8\right)$. Since any $\left(d H_{\alpha i_{1}}^{(s)} \ldots i_{s}\right)_{E^{p}}(a)$ is a linear combination of $\left(d H_{i}^{(\nu)}\right)_{E^{y}(a)}$ modulo $\left(d X^{1}\right)_{E^{p}}(a), \ldots,\left(d X^{n}\right)_{F^{2}}(a)$, we may replace (2.11) $\bmod (2.12)$ by

$$
\begin{aligned}
& \sum_{\lambda, k} t_{k i}^{\lambda}\left\langle\left(\frac{\partial}{\partial l_{k}^{\lambda}}\right)_{E^{p}(a)},\left(d H_{j}^{(\nu)}\right)_{E \nu^{\prime}(a)}\right\rangle \\
& \left(\begin{array}{l}
i=1, \ldots, p \\
\nu=0, \ldots, p-1, \quad j=1, \ldots, t_{\nu-1}
\end{array}\right)
\end{aligned}
$$

Let $R$ be the rank of (2.14) mod (2.13). Then it is sufficient to show that

$$
R+p(n-p)+\frac{p(p-1)}{2}(n-p)=\frac{p(p+1)}{2}(n-p)+t_{0}+2 t_{1}+\ldots+p t_{p-1},
$$

i.e.

$$
R=t_{0}+2 t_{1}+\ldots+p t_{p-1} .
$$

Let the variables $t_{k i}^{\lambda}$ in (2.14) be symmetric in the lower indices, i.e. $t_{k i}^{\lambda}=t_{i k}^{\lambda}$. Then $R$ is equal to the rank of the linear forms (2.14) of the variables $t_{k i}^{\lambda}=t_{i k}^{\lambda}$.

Since by the definition of $H_{j}^{(\nu)}($ see 8$\left.), \S 1\right) H_{j}^{(\nu)}$ are functions of $X^{1}, \ldots$, $X^{n}$ and $l_{k}^{\lambda}$ such that $k \leqq \nu,(2.14)$ may be written in the form

$$
\sum_{\lambda=p+1}^{n} \sum_{k=1}^{\nu} t_{k i}^{\lambda}\left\langle\left(\frac{\partial}{\partial l_{k}^{\lambda}}\right)_{F^{p}}(a), \quad\left(d H_{j}^{(\nu)}\right)_{E^{p}}(a)\right\rangle=A_{j i}^{(\nu)} .
$$

Since $\left\langle\left(\frac{\partial}{\partial l_{k}^{\lambda}}\right)_{F^{\nu}(a)},\left(d H_{j}^{(\nu)}\right)_{E^{n}(a)}\right\rangle=\left(\frac{\partial}{\partial l_{k}^{\lambda}} H_{j}^{(\nu)}\right)_{F^{\nu}(a)}$, it follows from a property of $\left(d H_{j}^{(\nu)}\right)_{E^{\prime}{ }^{\prime}(a)}$ mentioned in $\left.\S 1,8\right)$ that if $\sum_{j=1}^{t, \lambda^{j}} A_{j i}^{(\nu)}$ does not contain the variable $t_{\nu i}^{\lambda}$, then $\lambda^{j}=0$ for $j=1,2, \ldots, t_{\nu-1}$.

Now let $\Sigma$ be a set of $A_{j i}^{(\varkappa)}$ such that $1 \leqq i \leqq \nu, j=1, \ldots, t_{\nu-1}, \nu=1, \ldots, p$. $\Sigma$ consists of $t_{0}+2 t_{1}+\ldots+p t_{p-1}$ linear forms $A_{j i}^{(y)}$ and we show that these are linear independent. Suppose that an $A_{j_{0} i_{0}}^{(\nu)}$ in $\Sigma$ is a linear combination of the other elements of $\Sigma$ and let

$$
A_{j_{0} i_{0}}^{(\nu)}=\sum_{\mu, j, i} \lambda_{j i}^{(\mu)} A_{j i}^{(\mu)}
$$

Let $\mu_{1}$ be the highest value of $\mu$ such that $\lambda_{j i}^{(u)} \neq 0$. Suppose $\mu_{1}>\nu$ and let $i_{1}$ be the highest value of $i$ such that $\lambda_{j i}^{\left(\mu_{1}\right)} \neq 0$. Since $A_{j i}^{(\mu)}$ is a linear combination of the variable $t_{u v}^{\lambda}=t_{v u}^{\lambda}$ such that $u, v \leqq \mu$ and since in (2.15) the variables $t_{\mu_{1} i_{1}}^{\lambda}$ can appear only in the term $\sum_{j} \lambda_{j i_{1}}^{\left(\mu_{1}\right)} A_{j i_{1}}^{\left(\mu_{1}\right)}$, it follows that $\sum_{j} \lambda_{j i_{1}}^{\left(\mu_{1}\right)} A_{j i_{1}}^{\left(\mu_{1}\right)}$ does not contain the variables $t_{\mu_{1} i_{1}}^{\lambda}$ and hence $\lambda_{j i_{1}}^{\left(\mu_{1}\right)}=0 \quad\left(j=1,2, \ldots, t_{\mu_{1}-1}\right)$. Thus $\lambda_{j i}^{(\mu)}$ $=0$ for $\mu>\nu$. Then since in (2.15) the variables $t_{\nu i}^{\lambda}=t_{i \nu}^{\lambda}(\nu \geqslant i)$ appears only in the term $\sum_{j} \lambda_{j i}^{(\nu)} A_{j i}^{(\nu)}$ it follows as above that $\lambda_{j i}^{(v)}=0$. This is a contradiction since $A_{j_{0} i_{0}}^{(\nu)}$ contains the variables $t_{\nu i_{0}}^{\lambda}$. 
Next we show that any $A_{w v}^{(\mu)}$ is a linear combination of $A_{j i}^{(y)} \in \Sigma$ by induction on $/$. For simplicity we use the notations ()$_{0}$ and $\langle,\rangle_{0}$ instead of ()$_{n: "}^{\prime \prime}(a)$ and $\langle,\rangle_{F^{\prime \prime}(a)}$ respectively. Now let $\mu=1$. Then by the definition of $H_{k}^{(v)}$, there exists a $\theta_{\alpha}^{(1)} \in \Xi$ such that $H_{u l}^{(1)}=\left\langle L_{1}(r),\left(\theta_{\alpha}^{(1)}\right)_{r}\right\rangle$ and $\left\langle L_{v}(r),\left(\theta_{\alpha}^{(1)}\right)_{r}\right\rangle=H_{u l}^{(v)}$. Then

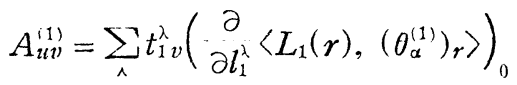

$$
\begin{aligned}
& =\sum_{\Lambda} t_{v_{1}}^{\lambda}\left\langle\left(\frac{\partial}{\partial x^{\lambda}}\right)_{a},\left(\theta_{\alpha}^{(1)}\right)_{a}\right\rangle \\
& =\sum_{\lambda} t_{v 1}^{\lambda}\left(\frac{\partial}{\partial l_{v}^{\lambda}}\left\langle L_{v}(r),\left(\theta_{\alpha}^{(1)}\right)_{r}\right\rangle\right)_{0} \\
& =A_{u 1}^{(v)} \text {. }
\end{aligned}
$$

Ilence $A_{u v}^{(1)} \in \Sigma$. Suppose that any $A_{u v}^{(u)}$ such that $\mu \leqslant \kappa-1$ is a linear combination of $A_{j i}^{(\nu)} \in \Sigma$. We take an $A_{u i}^{(\kappa)}$ such that $v>\kappa$. We firsi treat the case where $u \leqq t_{0}$. Then there exists a $\theta_{a}^{(1)} \in \cong$ such that $H_{u}^{(\alpha)}=\left\langle L_{\kappa}(r),\left(\theta_{\alpha}^{(1)}\right)_{r}\right\rangle$. Then

$$
\begin{aligned}
& \left\langle L_{v}(r),\left(\theta_{\alpha}^{(1)}\right)_{r}\right\rangle=H_{u}^{(1)}, \\
A_{u v v}^{(\kappa)}= & \sum_{\lambda} t_{\kappa \nu}^{\lambda}\left(\frac{\partial}{\partial l_{\kappa}^{\lambda}}\left\langle L_{\kappa}(r),\left(\theta_{\alpha}^{(1)}\right)_{r}\right\rangle\right)_{0} \\
= & \sum_{\Lambda} t_{v_{\kappa}}^{\lambda}\left\langle\left(\frac{\partial}{\partial x^{\lambda}}\right)_{a},\left(\theta_{\alpha}^{(1)}\right)_{a}\right\rangle \\
= & \sum_{\lambda} t_{v_{\kappa}}^{\lambda}\left(\frac{\partial}{\partial l_{v}^{\lambda}}\left\langle L_{v}(r),\left(\theta_{\alpha}^{(1)}\right)_{r}\right\rangle\right)_{0} \\
= & A_{u k \kappa}^{(v)} .
\end{aligned}
$$

Since $\kappa<v, A_{\imath \kappa}^{(v)} \in \Sigma$.

Nexi let $H_{u}^{(\kappa)}=\left\langle L_{i_{1}}(r)_{\wedge} \ldots \wedge L_{i_{3-1}}(r)_{\wedge} L_{\boldsymbol{k}}(r),\left(\theta_{\alpha}^{(S)}\right)_{r}\right\rangle$, where $1 \leqq i_{1}<\ldots$ $\left\langle i_{\text {s-1 }}<\kappa\right.$. Then $H_{u}^{(v)}=\left\langle L_{i_{1}}(r)_{\wedge} \ldots \wedge L_{i_{s-1}}(\boldsymbol{r})_{\wedge} L_{v}(r),\left(\theta_{x}^{(s)}\right)_{i}\right\rangle$.

Let

$$
\mathfrak{M}_{l}=\sum_{\lambda, k} t_{k l}^{\lambda}\left(\frac{\partial}{\partial l_{k}^{\lambda}}\right)_{0} \cdot \quad(l=1,2, \ldots, p)
$$

Then $A_{u v}^{(\kappa)}=\left\langle\mathfrak{M}_{v}, \quad\left(d H_{u}^{(\kappa)}\right)_{0}\right\rangle A_{u \kappa}^{(v)}=\left\langle\mathbb{M}_{\kappa}, \quad\left(d H_{u}^{(v)}\right)_{0}\right\rangle$. We show that

$$
\begin{gathered}
A_{w \nu}^{(\kappa)}-A_{i k \kappa}^{(v)}=\sum_{k=1}^{s-1}(-1)^{s-k}\left\langle\mathfrak{M}_{i_{k}},\right. \\
\left.d\left\langle L_{i_{\perp}}(r)_{\wedge} \ldots \wedge \widehat{L_{i_{k}}}(r)_{\wedge} \ldots \wedge L_{\kappa}(r)_{\wedge} L_{v}(r),\left(\theta_{\alpha}^{(s)}\right)_{r}\right\rangle_{v}\right\rangle,
\end{gathered}
$$

where the symbol $\wedge$ over $L$ indicates that the appropriate $L$ is to be omited.

$$
\begin{aligned}
A_{u v v}^{(\kappa)} & -A_{u \kappa}^{(p)} \\
& =\sum_{\lambda}\left(\sum_{k=1}^{s-1} t_{i_{k v} v}^{\lambda}\left(\frac{\partial}{\partial l_{i_{k}}^{\lambda}}\right)_{0}+t_{\kappa v}^{\lambda}\left(\frac{\partial}{\partial l_{\kappa}^{\lambda}}\right)_{0}\right), d\left\langle L_{i_{1}}(r)_{\wedge} \cdots \wedge L_{\kappa}(r),\left(\theta_{\alpha}^{(s)}\right)_{r}\right\rangle_{0} \\
& -\sum_{\Lambda}\left(\sum_{k=1}^{s-1} t_{i_{k \kappa}}^{\lambda}\left(\frac{\partial}{\partial l_{i_{k}}^{\lambda}}\right)_{0}+t_{v_{\kappa}}^{\lambda}\left(\frac{\partial}{\partial l_{v}^{\lambda}}\right)_{0}\right), d\left\langle L_{i_{1}}(r) \wedge \cdots \wedge L_{v}(r),\left(\theta_{\alpha}^{(s)}\right)_{r}\right\rangle_{0}
\end{aligned}
$$




$$
\begin{aligned}
& =\sum_{k=1}^{s-1}\left\langle\sum_{\lambda} t_{i_{k} v}^{\lambda}\left(\frac{\partial}{\partial l_{v}^{\lambda}}\right)_{0}\right. \\
& \left.d\left\langle L_{i_{1}}(r)_{\wedge} \cdots \wedge L_{i_{k-1}}(r)_{\wedge} L_{v}(r)_{\wedge} L_{i_{k+1}}(r)_{\wedge} \ldots \wedge L_{\kappa}(r),\left(\theta_{\alpha}^{(s)}\right)_{r}\right\rangle_{0}\right\rangle \\
& -\sum_{k=1}^{s-1}\left\langle\sum_{\lambda} t_{i k_{k}}^{\lambda}\left(\frac{\partial}{\partial l_{\kappa}^{\lambda}}\right)_{0}\right. \\
& \left.d\left\langle L_{i_{1}}(r) \wedge \cdots \wedge L_{i_{k-1}}(r)_{\wedge} L_{\kappa}(r)_{\wedge} L_{i_{k+1}}(r) \wedge \cdots \wedge L_{v}(r),\left(\theta_{\alpha}^{(s)}\right)_{r}\right\rangle_{\diamond}\right\rangle \\
& =\sum_{k=1}^{s-1}(-1)^{s-k}\left\langle\sum_{\lambda}\left\{t_{i_{k} v}^{\lambda}\left(\frac{\partial}{\partial l_{j}^{\lambda}}\right)_{0}+t_{i_{k} k}^{\lambda}\left(\frac{\partial}{\partial l_{k}^{\lambda}}\right)_{0}\right\},\right. \\
& \left.d\left\langle L_{i_{1}}(r)_{\wedge} \ldots \wedge \widehat{L_{i_{k}}}(r) \wedge \cdots \wedge L_{\kappa}(r)_{\wedge} L_{v}(r),\left(\theta_{\alpha}^{(s)}\right)_{r}\right\rangle_{0}\right\rangle \\
& =\sum_{k=1}^{s-1}(-1)^{s-k}\left\langle\mathfrak{M}_{i_{k}}, d\left\langle L_{i_{1}}(r)_{\wedge} \ldots \wedge \widehat{{i_{i k}}_{k}}(r)_{\wedge} \ldots \wedge L_{\kappa}(r)_{\wedge} L_{v}(r),\left(\theta_{\alpha}^{(s)}\right)_{r}\right\rangle_{0}\right\rangle \\
& -\sum_{k=1}^{s-1}(-1)^{s-k}\left\langle\sum_{\lambda} \sum_{l=1 \ldots s-1} t_{i \neq l}^{\lambda} i_{l \neq k}\left(\frac{\partial}{\partial l_{i}^{\Lambda}}\right)_{0},\right. \\
& \left.\left.d^{\prime} L_{i_{1}}(r)_{\wedge} \cdots \wedge \widehat{L_{i_{k}}}(r)_{\wedge} \cdots \wedge L_{\kappa}(r)_{\wedge} L_{v}(r),\left(\theta_{\alpha}^{(s)}\right)_{r}\right\rangle_{0}\right\rangle .
\end{aligned}
$$

Now the second term is zero. For, the coefficient of $t_{i_{i i_{k}}}^{\lambda}=t_{i_{k{ } l}}^{\lambda}$ is

$$
\begin{aligned}
& (-1)^{s-k}\left(\frac{\partial}{\partial l_{i_{l}}^{\lambda}}\left\langle L_{i_{1}}(r)_{\wedge} \ldots \wedge L_{i_{l}}(r) \wedge \cdots \wedge \widehat{L_{i_{k}}}(r)_{\wedge} \ldots,\left(\theta_{\alpha}^{(s)}\right)_{r}\right\rangle\right)_{0} \\
& +(-1)^{s-l}\left(-\frac{\partial}{\partial l_{i_{k}}^{\lambda}}\left\langle L_{i_{\iota}}(r) \wedge \ldots \wedge \widehat{L_{i l}}(r)_{\wedge} \ldots \wedge L_{i_{k}}(r)_{\wedge} \ldots,\left(\theta_{\alpha}^{(s)}\right)_{r}\right\rangle\right)_{0} \\
& =(-1)^{s-k} L_{i_{1}}(a)_{\wedge} \cdots \wedge\left(\frac{\partial}{\partial x^{\lambda}}\right)_{a} \wedge \cdots \wedge \widehat{L_{i_{k}}}(a)_{\wedge} \ldots,\left(\theta_{\alpha}^{(s)}\right)_{a} \\
& +(-1)^{s-l} L_{i_{1}}(a)_{\wedge} \ldots \wedge \hat{L_{i_{l}}}(a)_{\wedge} \ldots \wedge\left(\frac{\partial}{\partial x^{\lambda}}\right)_{a} \wedge \ldots,\left(\theta_{\alpha}^{(s)}\right)_{a}=0 .
\end{aligned}
$$

Now $\left\langle L_{i_{\perp}}(r) \wedge \cdots \wedge \hat{L_{i_{k}}}(r)_{\wedge} \ldots \wedge L_{\kappa}(r)_{\wedge} L_{v}(r),\left(\theta_{\alpha}^{(s)}\right)_{r}\right\rangle=H_{\alpha i_{1} \ldots \hat{i}_{k} \ldots \kappa v}^{(s)}$.

Let

$$
\left(d H_{\alpha i_{1} \ldots \hat{i}_{k} \ldots \kappa v}^{(S)}\right)_{0}=\sum_{j, \nu} C_{i_{1} \ldots \hat{i}_{k} \ldots i_{s-1}, j,}\left(d H_{j}^{(\nu)}\right)_{0}\left(\bmod .\left(d X^{1}\right)_{0}, \ldots\left(d X^{n}\right)_{0}\right)
$$

Then

$$
\begin{aligned}
& \left\langle\mathfrak{M}_{i_{i}}, d\left\langle L_{i_{1}}(r)_{\wedge} \cdots \wedge \widehat{L_{k}}(r)_{\wedge} \ldots \wedge L_{\kappa}(r)_{\wedge} L_{v}(r),\left(\theta_{\alpha}^{(s)}\right)_{r}\right\rangle_{0}\right\rangle \\
& =\sum_{j, \nu} C_{i_{\perp}} \ldots \hat{i_{k}} \ldots i_{s-1} j, A_{j i_{k}}^{(\nu)}
\end{aligned}
$$

If $i_{k} \leqq \nu$, then $A_{j i_{k}}^{(\nu)} \in \Sigma$. If $\nu<i_{k}$, then since $i_{k}<\kappa, A_{j i k_{k}}^{(\nu)}$ is a linear combination of $A_{j i}^{(\nu)} \in \mathbf{L}$ by the assumption of induction. We then see from (2.17) and (2.18) that $A_{w v}^{(\kappa)}$ is a linear combination of $A_{j i}^{(\nu)} \in \Sigma$. Thus Lemma 1 is completely proved.

Lemma 2, Let $V^{n}$ be a linear space of dimension $m$ over a field $K$ and $V^{\prime \prime}$ a subspace of $V^{m}$ of dimension $p$ and $V^{: m}$ the dual space of $V^{m}$. Let $g^{1}$, $\ldots, g^{s}$ be the elements of $V^{*^{m}}$ such that $\left\langle u, g^{i}\right\rangle=0(i=1, \ldots, s)$ for all 
$u \in V^{p}$ and let $f^{1}, \ldots, f^{p}$ be the elements of $V^{\star m}$ such that $f^{1}, \ldots, f^{p}$ are linearly independent on $V^{p}$. Denote by $V^{m-p}$ the subspace of $V^{m}$ of dimension $m-p$ consisting of all the elements $u \in V^{m}$ such that $\left\langle u, f^{i}\right\rangle=0(i=1,2, \ldots$, $p)$. Let $\eta^{\prime}$ be the identity mapping of $V^{m-p}$ into $V^{m}$. Then the rank of the elements $g^{1}, \ldots, g^{s}$ is equal to the rank of the elements ${ }^{t} \eta^{\prime}\left(g^{1}\right), \ldots,{ }^{t} \eta^{\prime}\left(g^{s}\right)$ of the linear space $V^{*^{m-p}}$, where ${ }^{t} \eta^{\prime}$ is the transposed mapping of $\eta^{\prime}$ and $V^{*^{m-p}}$ is the dual space of $V^{m-p}$.

This may be easily seen from the fact that $V^{m}$ is the direct sum of the subspaces $V^{p}$ and $V^{m-p}$.

Lemma 3. Let $E_{0}^{p}\left(a_{0}\right)$ be an integral element ${ }^{11)}$ of dimension $p$ of the differential system (1.2) on which $\left(d x^{1}\right)_{a}, \ldots,\left(d x^{p}\right)_{a}$ are linearly independent and let $E_{0}^{p}\left(E_{0}^{p}\left(a_{0}\right)\right)$ be an integral element of dimension $p$ and of origin $E_{0}^{p}(a)$ of the prolonged system (1.17), on which $\left(d X^{1}\right)_{E_{v}^{p}\left(a_{0}\right)}, \ldots,\left(d X^{p}\right)_{F_{0}^{p}\left(a_{0}\right)}$ are linearly independent. Further let $s_{0}, s_{1}, \ldots, s_{p-1}$ and $S_{0}, S_{1}, \ldots, S_{p-1}$ be the characters of $E_{0}^{p}(a)$ and $E_{0}^{p}\left(E_{0}^{p}(a)\right)$ respectively.

Then

$$
S_{1}+2 S_{2}+\ldots+p S_{p} \leqq \frac{p(p+1)}{2}(n-p)-\left(t_{0}+2 t_{1}+\ldots+p t_{p-1}\right),
$$

where $\quad S_{p}=(N-p)-\left(S_{0}+\ldots+S_{p-1}\right)$ and $t_{v}=s_{0}+\ldots+s_{v}$.

Proof. Let

$$
\begin{aligned}
& \mathscr{Q}_{i}^{0}=\left(\frac{\partial}{\partial X^{i}}\right)_{F_{0}^{p}\left(a_{0}\right)}+\sum_{\Lambda} t_{i}^{\lambda}\left(\mathbb{F}_{0}^{p}\right)\left(\frac{\partial}{\partial X^{\lambda}}\right)_{F_{v}^{p}\left(a_{0}\right)}+\sum_{\lambda, k} t_{k i}^{\lambda}\left(\mathscr{F}_{0}^{p}\right)\left(\frac{\partial}{\partial l_{k}^{\lambda}}\right)_{F_{0}^{p}\left(a_{0}\right)}, \\
& \mathfrak{S}_{i}=\left(\frac{\partial}{\partial X^{i}}\right)_{E_{0}^{p_{i}}\left(a_{0}\right)}+\sum_{\lambda} t_{i}^{\lambda}\left(\frac{\partial}{\partial X^{\lambda}}\right)_{E_{0}^{p_{i}}\left(a_{0}\right)}+\sum_{\lambda, k} t_{k i}^{\lambda}\left(\frac{\partial}{\partial l_{k}^{\lambda}}\right)_{E_{0}^{p}\left(a_{0}\right)},
\end{aligned}
$$

where $i=1,2, \ldots, p$ and $\mathfrak{C}_{0}^{p}=\mathfrak{C}_{0}^{p}\left(E_{0}^{p}\left(a_{0}\right)\right)$ and $t_{i}^{\lambda}, t_{k i}^{\lambda}$ are variables. Then $\left(\Omega_{1}^{0}, \ldots, \Omega_{p}^{0}\right)$ is a base of $\mathcal{F}_{0}^{p}$ and we denote by $\mathscr{E}_{0}^{\circ}$ the subspace of $\mathbb{E}_{0}^{p}$ spanned by $\mathfrak{R}_{1}^{0}, \ldots, \mathfrak{L}_{0}^{0}(\nu=1, \ldots, p-1)$ and by $\mathfrak{E}_{0}^{0}$ the origin $E_{0}^{p}\left(a_{0}\right)$ of $\mathfrak{E}_{0}^{p}$. Let $T\left(E_{0}^{\prime}\right)$ be the rank of the polar functions of the integral elements $\xi_{0}^{\prime}$ of the prolonged system $\widetilde{夭}$. We substitute $\Omega_{i}\left(E^{p}(r)\right)$ by $\Omega_{\nu}$ and $E^{p}(r)$ by $E_{0}^{p}\left(a_{0}\right)$ in (2.6), (2.7), and $(2.8)$ and $\mathfrak{S}_{i}\left(E^{p}(\boldsymbol{r})\right)$ by $\mathfrak{L}_{i}^{0}(i=1, \ldots, \nu-1), \mathfrak{Q}_{j}\left(E^{p}(\boldsymbol{r})\right)$ by $\mathfrak{L}_{\nu}$ and $E^{p}(r)$ by $E_{0}^{p}\left(a_{0}\right)$ in (2.9) respectively. Then we obtain a system of linear equations of the variables $t_{\nu}^{\lambda}, t_{k \nu}^{\lambda}(\lambda=p+1, \ldots, n ; k=1, \ldots, p)$. Applying Lemma 2 in the case where $m=N, \quad V^{m}=F^{*}\left(E_{0}^{p}\left(a_{0}\right)\right)=\mathfrak{F}^{N}, V^{p}=\mathbb{E}_{0}^{p}, \quad f^{i}$ $=\left(d X^{i}\right)_{E_{0}^{v}\left(a_{0}\right)}$ and $g^{1}, \ldots, g^{s}$ are the polar functions of $E_{0}^{2}$ and considering the fact that the space $F^{N-p}$ consisting of all the elements $\mathbb{L} \in \mathfrak{F}^{N}$ such that $<\mathbb{R}$, $\left.\left(d X^{i}\right)_{E_{0}^{\nu}(a)}\right\rangle=0(i=1, \ldots, p)$ is spanned by $\left(\frac{\partial}{\partial X^{\Lambda}}\right)_{E_{0}^{p}\left(a_{0}\right)}$ and $\left(\frac{\partial}{\partial l_{k}^{\lambda}}\right)_{E_{0}^{p}\left(a_{0}\right)}(\lambda=$ $p+1, \ldots, n ; k=1, \ldots, p)$, we see that the rank of this system of linear

11) We do not assume here that $E_{0}^{p}\left(a_{0}\right)$ is ordinary. 
equations of the variables $t_{\nu}^{\lambda}, t_{k \nu}^{\lambda}$ is equal to $T\left(\xi_{0}^{\nu-1}\right) \quad(\nu=1,2, \ldots, p)$. Now the homogeneous parts of these equations are

$$
\begin{aligned}
& \sum_{\lambda} t_{\nu}^{\lambda}\left\langle\left(\frac{\partial}{\partial X^{\lambda}}\right)_{E_{0}^{p}\left(a_{0}^{\prime \prime},\right.}\left(d H_{\alpha}^{(0)}\right)_{F_{a}^{p}\left(a_{0}\right)}\right\rangle \quad\left(\alpha=1, \ldots, u_{0}\right) . \\
& \sum_{\lambda} t_{\lambda}^{\lambda}\left(\frac{\partial}{\partial X^{\wedge}}\left\langle L_{i_{1}}(r) \wedge \cdots \wedge L_{i_{s}}(r),\left(\theta_{\alpha}^{(s)}\right)_{r}\right\rangle\right)_{E_{0}^{p}\left(a_{0}\right)} \\
& +\sum_{\wedge} \sum_{k=1}^{s} t_{i_{k} \nu}^{\lambda}\left(\frac{\partial}{\partial l_{i_{k}}^{\lambda}}\left\langle L_{i_{1}}(r)_{\wedge} \ldots \wedge L_{i_{s}}(r),\left(\theta_{\alpha}^{(s)}\right)_{r}\right\rangle\right)_{F_{0}{ }^{p}\left(a_{0}\right)} \\
& \left(\alpha=1, \ldots, u_{s}, \quad s=1,2, \ldots, \quad 1 \leqq i_{1}<\ldots<i_{s} \leqq p\right)
\end{aligned}
$$

Now let

$$
\begin{aligned}
\sum_{\wedge} \sum_{k=1}^{s} t_{i_{k} \times}^{\lambda}\left(\frac{\partial}{\partial l_{i_{k}}^{\lambda}}\left\langle L_{i_{1}}(r) \wedge \ldots \wedge L_{i_{s}}(r),\left(\theta_{\alpha}^{(s)}\right)_{r}\right\rangle\right)_{F_{0}{ }_{0} p_{\left(a_{0}\right)}} & \left(\alpha=1, \ldots, u_{s}, \quad s=1,2, \ldots, \quad 1 \leqq i_{1}<\ldots<i_{s} \leqq p\right)
\end{aligned}
$$

We derote by $R_{v}$ the number of the linear forms in (2.23) which are linearly independent $\bmod$ (2.22). Then $R_{\nu}+(n-p)+(\nu-1)(n-p)=T\left(\xi_{0}^{\prime-1}\right)$. Now we consider the linear forms in $(2.23)$ such that $i_{s}=\nu$. Since $i_{1}<\ldots<i_{s-1}<\nu$

$$
\begin{aligned}
& \left.\sum_{\wedge} \sum_{k=1}^{s} t_{i_{k},}^{\lambda}\left(\frac{\partial}{\partial l_{i_{k}}^{\lambda}}\left\langle L_{i_{1}}(\boldsymbol{r})_{\wedge} \ldots \wedge L_{i_{s}}(\boldsymbol{r}),(\theta)_{\alpha}^{(s)}\right)_{r}\right\rangle\right)_{E_{0}^{p}(\boldsymbol{a})} \\
& \equiv \sum_{\wedge} t_{\nu 2}^{\lambda}\left(\frac{\partial}{\partial l_{v}^{\lambda}}\left\langle L_{i_{1}}(r) \wedge \cdots \wedge L_{\nu}(r),\left(\theta_{\alpha}^{(s)}\right)_{r}\right\rangle\right)_{E_{0}^{p}(a)} \\
& \equiv\left\langle L_{i_{1}}^{0}\left(a_{0}\right) \wedge \cdots \wedge L_{i_{s-1}}^{0}\left(a_{0}\right) \wedge\left(\sum_{\wedge} t_{\nu_{\nu}}\left(\frac{\partial}{\partial x^{\lambda}}\right)_{a_{0}}\right),\left(\theta_{\alpha}^{(s)}\right)_{a_{0}}\right\rangle(\bmod (2.22)) \text {, }
\end{aligned}
$$

where $L_{k}^{0}\left(a_{0}\right)=\left(\frac{\partial}{\partial x^{k}}\right)_{a_{0}}+\sum_{\lambda=p+1}^{n} l_{k}^{\lambda}\left(E_{0}^{p}\left(a_{0}\right)\right)\left(\frac{\partial}{\partial x^{\lambda}}\right)_{a_{0}}(k=1, \ldots, p)$ forms a base of $E_{0}^{p}\left(a_{0}\right)$. Let $E_{0}^{\iota}\left(a_{0}\right)$ be the subspace of $E_{0}^{p}\left(a_{0}\right)$ spanned by $L_{1}^{0}\left(a_{0}\right), \ldots, L_{0}^{0}\left(a_{0}\right)$. If necessary replacing $x^{1}, \ldots, x^{p}$ by suitable linear combinations of them with constant coefficients, we may assume that $E_{0}^{\nu}\left(a_{0}\right)(\nu=0, \ldots, p-1)$ have the properties that $t_{2}=s_{0}+\ldots+s_{\nu}=t\left(E_{0}^{\nu}(a)\right)$. Applying Lemma 2 in the case where $m=n, V^{m}=E^{n}\left(a_{0}\right), \quad V^{p}=E_{0}^{p}\left(a_{0}\right), f^{i}=\left(d x^{i}\right) a_{0}$ and $g^{i}, \ldots, g^{s}$ are the polar functions of $E_{0}^{\prime}\left(a_{0}\right)$ and considering that the subspace $E^{n-p}\left(a_{0}\right)$ consisting of all the elements $L \in E^{n}\left(a_{0}\right)$ such that $\left\langle L,\left(d x^{2}\right)_{a_{0}}\right\rangle=0(i=1, \ldots, p)$ is spanned by $\left(\frac{\partial}{\partial x^{\lambda}}\right)_{a_{0}}(\lambda=p+1, \ldots n$, we see that the rank of the linear forms of the variables $t_{i}^{i}$ which are obtained above is equal to the rank of the polar functions of $E_{0}^{v-1}$ which is equal to $s_{0}+\ldots+s_{\nu-1}$. Hence we obtain $s_{0}+\ldots$ $+s_{\nu-1}$ linear forms in (2.22) which are linearly independent mod (2.22). Next we consider the linear forms in (2.23) such that $i_{s}=\nu+1$. Then we can show 
as above that these are reduced mod (2.22) to the forms

$$
\left\langle L_{i_{1}}^{0}\left(a_{0}\right)_{\wedge} \ldots \wedge L_{i_{s-1}}^{0}\left(a_{0}\right) \wedge \sum_{\lambda} t_{\nu+1}^{\lambda}\left(\frac{\partial}{\partial x^{\lambda}}\right)_{a_{0}},\left(\theta_{\alpha}^{(s)}\right)_{a_{0}}\right\rangle,\left(i_{1}<\ldots<i_{s-1}<\nu\right),
$$

or

$$
\begin{aligned}
& \left\langle L_{i_{1}}^{0}\left(a_{0}\right) \wedge \cdots \wedge L_{i_{s-2}}^{0}\left(a_{0}\right) \wedge\left(\sum_{\lambda} t_{\nu}^{\lambda \nu}\left(\frac{\partial}{\partial x^{\lambda}}\right) a_{0}\right) \wedge L_{\nu+1}^{0}\left(a_{0}\right),\left(\theta_{\alpha}^{(s)}\right)_{a_{0}}\right\rangle \\
+ & \left\langle L_{i_{1}}^{0}\left(a_{0}\right) \wedge \cdots \wedge L_{\nu}^{0}\left(a_{0}\right)_{\wedge}\left(\sum_{\lambda} t_{\nu+1 \nu}^{\lambda}\left(\frac{\partial}{\partial x^{\lambda}}\right)_{a_{0}}\right),\left(\theta_{\alpha}^{(s)}\right)_{a_{0}}\right\rangle,\left(i_{1}<\ldots<i_{s-2}<\nu\right),
\end{aligned}
$$

according as $i_{s-1}<\nu$ or $i_{s-1}=\nu$. The rank of the linear forms in the variables $t_{\nu+1 \nu}^{\lambda}$ which appear in the above linear forms is equal to the rank of the polar functions of $E_{0}^{\prime}(a)$ which is equal to $s_{0}+\ldots+s_{w}$. Hence we obtain $s_{0}+\ldots$ $+s_{v}$ linear forms in (2.23) which are linearly independent mod (2.22) and are linearly independent $\bmod (2.22)$ to $s_{0}+\ldots+s_{v-1}$ linear forms already obtained. Continuing these considerations, we obtain

$$
\begin{aligned}
R_{\nu}^{\prime} & =\left(s_{0}+\ldots+s_{\nu-1}\right)+\left(s_{0}+\ldots+s_{v}\right)+\ldots+\left(s_{0}+\ldots+s_{p-1}\right) \\
& =t_{\nu-1}+\ldots+t_{p-1}
\end{aligned}
$$

linear forms in (2.23) which are lineariy independint $\bmod (2.22)$. Hence $R_{\nu}$ $\Rightarrow R_{v}^{\prime}$ and therefore we get the inequality

$$
T\left(\xi_{0}^{\nu-1}\right) \geqslant \nu(n-p)+i_{v-1}+\ldots+i_{p-1} .
$$

Now let $\widetilde{S}_{0}=T\left(\xi_{0}^{0}\right), \widetilde{S}_{\nu}=T\left(\xi_{0}^{\nu}\right)-T\left(\xi_{0}^{\nu-1}\right) \quad(\nu=1,2, \ldots, p-1)$ and $\widetilde{S}_{p}$ $=(N-p)-\left(\widetilde{S}_{0}+\ldots+\widetilde{S}_{p-1}\right)$. Then

$$
\widetilde{S}_{1}+2 \widetilde{S}_{2}+\ldots+p \widetilde{S_{p}}=p(N-p)-\left(T\left(\xi_{0}^{0}\right)+\ldots+T\left(\xi_{0}^{p-1}\right)\right) .
$$

In the same way

$$
S_{1}+2 S_{2}+\ldots+p S_{p}=p(N-p)-\left(T_{0}+\ldots+T_{p-1}\right),
$$

where $T_{\nu}=S_{0}+\ldots+S_{\nu}=\underset{\mathscr{E}^{2} \subset \mathbb{C}^{p}}{\operatorname{Max}} T\left(\xi^{2}\right) \geq T\left(\xi_{0}^{\nu}\right)$. Hence

$$
S_{1}+2 S_{2}+\ldots+p S_{p} \leqq \widetilde{S}_{1}+2 \widetilde{S}_{2}+\ldots+p \widetilde{S}_{p} .
$$

From (2.24) and (2.25) we get

$$
\begin{aligned}
\widetilde{S}_{1}+2 \widetilde{S}_{2}+\ldots+p \widetilde{S}_{p} \leqq p(N-p) & -(n-p) \frac{p(p+1)}{2} \\
& -\left(t_{0}+2 t_{1}+\ldots+p t_{p-1}\right) .
\end{aligned}
$$

Since $N=n+(n-p) p$, we obtain

$$
S_{1}+2 S_{2}+\ldots+p S_{p} \leqq \frac{p(p+1)}{2}(n-p)-\left(t_{0}+2 t_{1}+\ldots+p t_{p-1}\right) .
$$

q.e.d.

Now we show that the rank $R$ of the linear forms $(2.10), \ldots,(2.13)$ is 
equal to $p S_{0}+(p-1) S_{1}+\ldots+S_{p-1}$. Let us consider the differentials at the point $E_{0}^{p}=E_{0}^{p}\left(E_{0}^{p}\left(a_{0}\right)\right)$ of the functions which appear in the left sides of the equations (2.6), . ., (2.9). Applying the result of 8$), \S 1$ in our case, we see readily that there exist at least $p S_{0}+(p-1) S_{1}+\ldots+S_{p-1}$ ones among these differentials which are linearly independent modulo $d\left(X^{i} \circ \Pi\right)_{\mathfrak{E}_{0}^{p}}, \quad(i=1, \ldots, p)$, $d\left(l_{k}^{\lambda} \circ I\right) \mathfrak{S}_{0}^{p}(\lambda=p+1, \ldots, n, k=1, \ldots, p)$. If we replace in the linear forms $(2.10), \ldots,(2.13) t_{i}^{\lambda}, t_{i j}^{\lambda}$ and $E^{p}(a)$ by $\left(d t_{i}^{\lambda}\right) \mathfrak{E}_{v}^{v}$, $\left(d t_{i j}^{\lambda}\right) \mathfrak{E}_{0}^{p}$ and $E_{0}^{p}\left(a_{0}\right)$ respectively, we obtain linear expressions in $\left(d t_{i}^{\lambda}\right)_{\mathfrak{E}_{0}^{p}},\left(d t_{i j}^{\lambda}\right)_{\mathbb{E}_{0}^{p}}$ which are equal to the differentials at the point $\mathbb{E}_{0}^{p}$ which we have considered above. Hence if we apply Lemma 1 in the case $E^{p}(a)=E_{0}^{p}\left(a_{0}\right)$, we get $p S_{0}+(p-1) S_{1}+\ldots+S_{p-1}$ $\leqq \frac{p(p+1)}{2}(n-p)+t_{0}+2 t_{1}+\ldots+p t_{p-1}$. On the other hand we get by Lemma $3, p(N-p)-\left(S_{1}+2 S_{2}+\ldots+p S_{p}\right) \Rightarrow \frac{p(p+1)}{2}(n-p)+t_{0}+2 t_{1}+\ldots$ $+p t_{p-1}$, hence $p S_{0}+(p-1) S_{1}+\ldots+S_{p-1} \geqslant \frac{p(p+1)}{2}(n-p)+t_{0}+2 t_{1}+\ldots$ $+p t_{p-1}$. Thus we obtain the equality $p S_{0}+(p-1) S_{1}+\ldots+S_{p-1}=\frac{p(p+1)}{2}(n$ $-p)+t_{0}+2 t_{1}+\ldots+p t_{p-1}$. Hence by Lemma $1 R=p S_{0}+(p-1) S_{1}+\ldots$ $+S_{p-1}$. Thus $\left(_{0}^{p}\right.$ is ordinary.

Now it follows from $(2.27)$ and $(2.28)$ that $\widetilde{S}_{1}+2 \widetilde{S}_{2}+\ldots+p \widetilde{S}_{p}=S_{1}+2 S_{2}$ $+\ldots+p S_{p}$. Hence we get from (2.25) and (2.26) the equalties $T_{\nu}=T\left(5_{0}^{2}\right)$ $(\nu=0,1, \ldots, p-1)$. Since it holds the equality in $(2.28)$, it must hold also the equalities in (2.24). Therefore we have

$$
S_{0}+\ldots+S_{\nu}=T_{\nu}=(\nu+1)(n-p)+t_{\nu}+\ldots+t_{p-1} .
$$

Then we have, $S_{\nu}=(n-p)-t_{\nu-1}=s_{\nu}+\ldots+s_{p}$. Thus Theorem 1 is completely proved.

\section{REFERENCES}

[1] Bourbaki, N., Algèbre, Chapiter II, Algèbre Linéaire, Chapitre III, Algèbre Multilinéaire, 1948.

[2] Cartan, E., Sur la structure des groupes infinis de transformations, Annales École normale sup., 21, 1904.

[3] Cartan, E., Les systèmes différentiels extérieurs et leurs applications géométriques, Paris, 1945.

[4] Chevalley, C.. Theory of Lie Groups. I, Princeton, 1946.

[5] Kähler, E., Einführung in die Theorie der Systeme von Differentialgleichungen, Hamburger Math. Einzelschrifte, 16, 1934.

[6] Schouten, J. A. and van der Kulk, W., Pfaff's problem and its generalizations, Oxford, 1949.

Mathematical Institute, Nagoya University 